\title{
Removal of ECG Baseline Wander using Savitzky-Golay Filter Based Method
}

\author{
K M Talha Nahiyan and Abdullah-Al Amin \\ Department of Biomedical Physics and Technology, University of Dhaka \\ Email: tnahiyan@du.ac.bd, a.amin@bmpt.du.ac.bd
}

\begin{abstract}
ECG (Electrocardiogram) is a measure of heart's electrical activity. As the body is a volume conductor, ECG signal can be recorded from the body surface. The signal while being recorded from the body surface gets corrupted by various types of noise or artifact. Among these, baseline wander is a type of noise that severely hampers the ECG signal. Baseline wander is particularly of very low frequency; it causes the ECG signal to deviate from its isoelectric line and causes the signal to ride on the lower frequency artifact. The proposed method is based on Savitzky-Golay filter, which is a moving average filter that takes into consideration the polynomial order along with moving averaging when approximating the signal. It enables to approximate the baseline wander quite efficiently. Though in some cases it distorts the ECG signal to some extent, when compared with usual polynomial fitting method, it demonstrates superiority in terms of accuracy, simplicity and generalization.
\end{abstract}

Keywords: Electrocardiogram (ECG), Baseline Wander Removal, Savitzky-Golay Filter.

\section{INTRODUCTION}

ECG signal is affected by various types of artifact even if the signal is acquired very carefully. The most significant of these are baseline wander, muscle artifact (EMG) and power line interference. The shift or change in the ECG isoelectric line or baseline is characterized as baseline wander (BW). It is a type of noise that does not distort the ECG signal as much as muscle artifact or powerline interface but is more difficult to remove. The primary source of BW is respiration, more precisely by the spatial changes caused by lung movements. It can also generate from body movement such as in case of a restless patient, which changes the electrode contact potential (Akay, 2006). Perspiration is also known to cause change in electrode impedance thus introducing possible BW(Macfarlane, 2011). Removal of BW is especially important when analyzing low frequency ST segments for detecting ischemia during stress tests (Akay, 2006). The BW noise spectrum is usually localized in low frequencies. Usually it is less than $0.5 \mathrm{~Hz}$ (Macfarlane, 2011). However, increased movement of the body during the latter stages of a stress test further increases the frequency content of BW. It is essential to determine the cutoff frequency of the BW based on ECG signal frequency band. The lowest heart rate that can drop during bradycardia is $40 \mathrm{bpm}$ (Akay, 2006). According to that, in their 1990 recommendations, the AHA (American Heart Association) stipulated that a BW removal filter should have a flat amplitude response within $0.5 \mathrm{~dB}$ from 1 to $30 \mathrm{~Hz}$, with a $-3 \mathrm{~dB}$ cutoff frequency of less than $0.67 \mathrm{~Hz}$ (Bailey et al., 1990). It also recommends testing certain criteria based on triangular and rectangular waveform pulses (Bailey, 2004). Another fact that needs to be taken into consideration is that the frequency range of the amplifier for diagnostic ECG is $0.05-150 \mathrm{~Hz}$. Thus, the frequency spectrum of BW and ECG signal amplifier overlaps to some extent (Webster, 1988).

\section{BACKGROUND}

Different techniques have been used over time to remove BW from ECG signal. The earliest techniques used are based on polynomial fitting. BW estimation and removal using cubic spline method is demonstrated by Meyer and Keiser (1977). Further improvements on this can be found in work by Badilini et al. (1991). In this work cubic spline technique estimates a continuous baseline by joining 
isoelectric points (fiducial points) with third order lines (splines). The parameters of the various splines are calculated by matching first and second derivatives in the inner fiducial points thus determining a resulting smoothed line. It provides reliable measurement of ST segment displacements leading to automatic detection of myocardial ischemia. But these methods can break down if the knot selection assumption for polynomial fitting is not met due to premature beat merging or heart rate variability (Froning, et al., 1988).

Another widely used technique involves implementation of high pass digital filters. The filters need to have linear phase and minimal distortion. Thus infinite impulse response (IIR) filters are generally unacceptable due to their nonlinear phase response. However, in offline processing or considering the delay in responses allows acceptable results. Forward-backward or bidirectional filtering can be applied to get such linear phase results (Pottala et al., 1990) (Frankel et al., 1991). An alternative method of using linear phase low pass filter was proposed by De Pinto (1992). The output of the low pass IIR filter was subtracted from the original signal to obtain a BW free signal. Though linear phase filtering can be achieved easily using FIR filters but as they tend to have long impulse responses, it leads to very high filter order with complex multiplications (Frankel et al., 1991; Van Alste and Schilder, 1985).

Jane et al. (1992) described a two stage cascaded adaptive filter which consists of an adaptive notch filter at zero frequency at first stage and an adaptive impulse correlated filter in second stage. It works in real time and comparably worked better than cubic spline methods.

Wavelet transform has also been applied for removal of BW. Park et al. (1998) described a wavelet adaptive filter. The filter consists of two parts. A wavelet transform decomposes the ECG signal into seven frequency bands. The signal of the lowest frequency band is then fed into an adaptive filter. Performance of this filter was better than standard high pass filters and adaptive filters.

All techniques already discussed above have a priori basis and are not particularly suitable for nonlinear or non-stationary signals. Blanco-Velasco et al. (2008) proposed a method for BW based on empirical mode decomposition (EMD). Empirical mode decomposition was originally developed by Huang et al. (1998) as a part of Hilbert-Huang transform (HHT) for analysis of nonlinear and non-stationary signals. The EMD relies on a fully data-driven mechanism that does not require any a priori known basis. The aim of the EMD is to decompose the signal into a sum of intrinsic mode functions (IMFs). An IMF is defined as a function with equal number of extrema and zero crossings (or at most differed by one) with its envelopes, as defined by all the local maxima and minima, being symmetric with respect to zero. An IMF represents a simple oscillatory mode as a counterpart to the simple harmonic function used in Fourier analysis. Since BW is a low-frequency phenomenon, it is expected that the major BW components are located in the higher-order IMFs. Simply removing the last several IMFs may introduce significant distortions. Thus, the BW is separated from the desired components in the last several IMFs. To remove the BW, an estimate is first obtained via a "multiband" filtering approach and then subtracted from the original signal. The method shows better performance than standard digital filter or wavelet based filters for real BW (Blanco-Velasco et al., 2008).

\section{PROBLEM STATEMENT}

The standard digital filters both IIR and FIR have implications which do not allow efficient estimation of BW as it tends to distort the original ECG signal. As the BW frequency range tends to overlap with the ECG signal frequency range, it is likely that such distortions would be inevitable if digital filters are used. Using polynomial fitting needs selecting of polynomial order properly and the order may not be 
appropriate for all cases. Moreover higher orders would need more complex calculation and cause ringing effect on estimated $\mathrm{BW}$, which would distort the ECG signal significantly. Wavelet transform based methods do perform better but the assumptions made are all priori and does not serve an adaptive approach. EMD could provide an empirical, nonlinear signal processing method which could be appropriate for ECG signal processing as can be seen in work by Blanco-Velasco et al. But their approach of removing BW from the intrinsic mode functions (IMF) using a low pass filtering approach seems to contradict with the reason for moving away from standard digital filters and also the method is not tested against AHA recommended triangular wave or square wave which represents abrupt baseline shift.

Moving average filter is one of the easiest kinds of filter to implement as it can be applied on time domain signal. Transforming the signal to frequency domain is not needed. Savitzky-Golay (SG) filter being a kind of moving average filter demonstrates similar characteristics. It can be applied on time domain signal and does not have any parameter dependent on frequency domain. For this, it is also suitable for processing ECG signal. In this way, it does not affect the ECG signal portions that have overlapping frequency spectrum with BW. The method would work on ECG signal irrespective of frequency spectrum and length of the signal. Also no prior knowledge of the artifact's attributes is required. Analysis of properties of SG filter from the ECG signal processing aspect was done by Hargittai (Hargittai, 2005). Zheng et al. (Zheng, Lall and Chen, 2012) using a combination of FIR low pass filter and SG filter to remove BW. The proposed method in this paper for removing BW is based on a combination of SG filter and moving average filter. Further explanation on appropriateness of using SG filter is given later.

\section{METHODS}

It is necessary to divide the ECG signal into short windows and to process conveniently. The ECG signal would not have an assigned length when working with real cases. For various feature extraction purposes different lengths of ECG signal may be necessary, such as for analyzing an ECG stress test, about half-hour length data may be needed to process. Also more importantly, selecting a convenient short window would allow to minimize the chance of different trends in a data length. Suppose, two different types of trend can be approximated similarly, over a short window but for whole data length that might not be possible.

The algorithm for estimating the BW can be divided into two distinct steps. Firstly in every window of the ECG signal, SG filter with polynomial degree of 1 is applied. The length of the filter for those points is kept close to the window length. It would then give an estimated output in every window, which is a slope preserving the trend of the signal. This step is applied for each window and when all the outputs are added gives a rough view of the trend.

The output from the first step will have sharp edges on the end and start of a window. Moreover the output only gives a slope type approximation. In this second step applying simple moving average filtering on the output will give a smoothed out, close approximation of the BW. After that simply subtracting the estimated BW from the original signal would give a baseline corrected ECG signal. The proposed approach for removal of BW would need a basic understanding of SG filter and simple moving average filter. So, before proceeding to details of the proposed method the theory of SG filter and moving average filter is discussed briefly. 
Savitzky-Golay Filter: Savitzky and Golay proposed a method of data smoothing based on local leastsquares polynomial approximation (Savitzky and Golay, 1964). They showed that fitting a polynomial to a set of input samples and then evaluating the resulting polynomial at a single point within the approximation interval is equivalent to discrete convolution with a fixed impulse response. The lowpass filters obtained by this method are widely known as Savitzky-Golay filters. Savitzky and Golay were interested in smoothing noisy data obtained from chemical spectrum analyzers, and they demonstrated that least squares smoothing reduces noise while maintaining the shape and height of waveform peaks (Schafer, 2011).

A good theoretical base for SG filter can be found in the book by Orfanidis (1996). It is presented here in a simple way. For a data vector $\mathbf{X}$ having $N$ data points ( $N$ odd, say $N=2 M+1$ ), there are $\mathrm{M}$ points on either side of $x_{0}$ :

$$
X=\left[x_{-M}, \ldots, x_{-1}, x_{0}, x_{1}, \ldots, x_{M}\right]^{T}
$$

The $N$ data samples in $\mathbf{X}$ are then fitted by a polynomial of degree $d$ :

$$
\hat{x}_{m}=c_{0}+c_{1} m+\cdots+c_{d} m^{d},-M \leq m \leq M
$$

In this case, there are $d+1$ polynomial basis vectors $\mathbf{s}_{\mathbf{i}}, i=0,1, \ldots, d$, is defined to have components:

$$
s_{i}(m)=m^{i}, \quad-M \leq m \leq M
$$

The corresponding $N^{*}(d+1)$ matrix $\mathbf{S}$ is defined to have $\mathbf{S}_{\mathbf{i}}$ as columns:

$$
S=\left[s_{0}, s_{1}, \ldots, s_{d}\right]
$$

The smoothed values (1b) can be written in the vector form:

$$
\hat{X}=\sum_{i=0}^{d} c_{i} s_{i}=\left[s_{0}, s_{1}, \ldots, s_{d}\right]\left[\begin{array}{l}
c_{0} \\
c_{1} \\
\vdots \\
c_{d}
\end{array}\right]=\mathrm{Sc}
$$

The design steps for the SG filters then can be summarized as follows as given in (Orfanidis, 1996):

$$
\begin{gathered}
F=S^{T} S \\
G=S F^{-1} \\
B=G S^{T}=S G^{T}=S F^{-1} S^{T} \equiv\left[b_{-M}, \ldots, b_{0}, \ldots, b_{M}\right]
\end{gathered}
$$

Here the components of $B$ are SG filters of length $N$ and order $d$. Then the corresponding smoothed data vector will be:

$$
\hat{X}=B X \leftrightarrow \hat{x}_{m}=b_{m}^{T} X, \quad-M \leq m \leq M
$$

The resulting length $N$, order $d$, SG filter for smoothing a noisy sequence $x(n)$ will be, in steady state form:

$$
y(n)=\sum_{m=-M}^{M} b_{0}(m) x(n+m)=\sum_{m=-M}^{M} b_{0}(-m) x(n-m) \quad \ldots(1 \mathrm{e})
$$

Simple Moving Average Filter: The moving average filter performs averaging a number of points from the input signal to produce each point in the output signal (Smith, 2002). It can be expressed as:

$$
y[i]=\frac{1}{M} \sum_{j=-(M-1) / 2}^{(M-1) / 2} \quad x[i+j]
$$


where $\mathrm{M}$ is the number of points used in the moving average. In this case the input points are chosen symmetrically around the output point thus $\mathrm{M}$ must be odd.

Selecting window for ECG signal processing: As discussed earlier that ECG BW spectrum usually less than $0.5 \mathrm{~Hz}$ (Macfarlane, 2011), the consideration of window length would be dependent on that. For any periodic nature of the trend, one-fourth cycle of that trend would appear within 0.5 second in time domain (considering $0.5 \mathrm{~Hz}$ as the threshold of BW trend). Thus a window length approximately close to 0.5 second in time domain would serve ideal. As the method is not frequency dependent rather is applied in time domain, the window size selected serves as a convenient length and it is not expected to undermine the method in case of BW trend frequency slightly varying the $0.5 \mathrm{~Hz}$ mark.

Selecting length and order of SG filter: The length $(N)$ of the SG filter should be odd and the length selected in each window is kept almost close to the window length but shorter than it. As a result, the output can be obtained containing the trend when order of the filter $(d)$ is selected as 1 . The linear order SG filtering on short windows only gives an estimation of the trends presence but not the actual trend. Also due to windowing the edges of the window are not smooth. Both these implications can be overcome in the next step.

Applying moving average filter: From the estimated presence of trend, the BW can be visualized by applying a moving average filter on the estimated data. In this case, the length of the moving average filter is chosen approximately $5 \%$ of the whole data length to preserve the shape of the trend. The moving average filtering has been repeated three times as otherwise there remain unwanted peaks at the edge of the windows. As the moving average smoothing is done on estimated BW, it has no effect on the high frequency components of the original ECG signal.

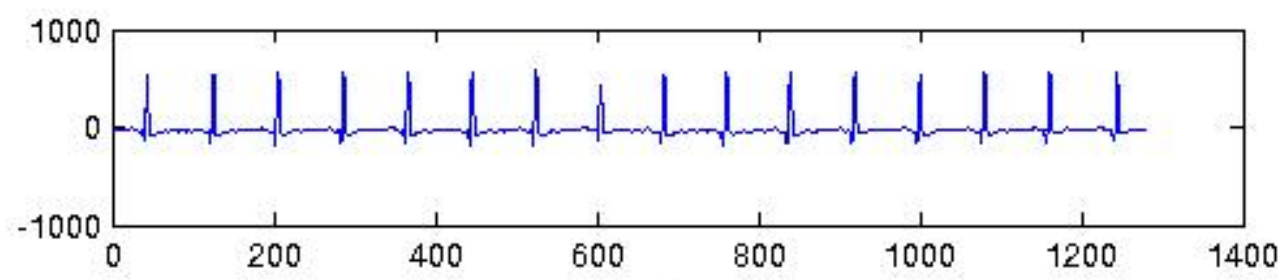

(a)

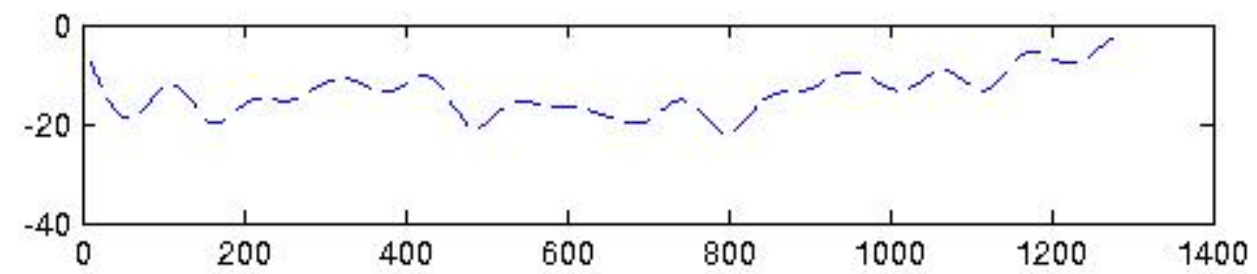

(b)

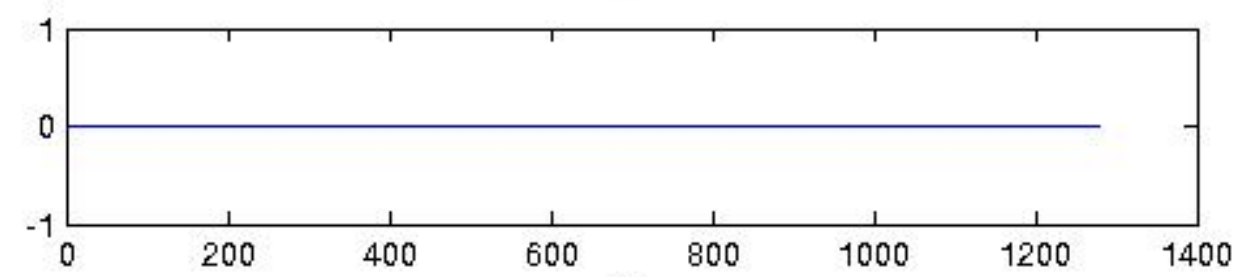

(c)

Fig. 1: (a) ECG signal without BW (b) Estimation without applying threshold condition (c) Estimation after applying threshold condition 
Setting condition for deciding true baseline wander: There would be situations where the ECG does not contain any BW. But the estimated data would have some approximation which is actually not any BW but just the combined first order polynomial trend of the original ECG data. In those cases the estimated BW would rather distort the ECG signal unnecessarily. The unwanted effect of such estimation is shown in fig.1. The remedy could be setting a threshold value to determine true cases of $\mathrm{BW}$. The standard deviation before and after moving average filtering could be an indicator to this. The standard deviation would definitely decrease after moving average filtering. The condition is set such that if the second standard deviation is lower than a certain percentage of first standard deviation then the estimation is rejected and is set to zero, denoting that no BW is present in the ECG signal. Experimentally this percentage is set $50 \%$ of the first standard deviation.

Algorithm in MATLAB ${ }^{\circledR}$ : For implementing SG filter, MATLAB ${ }^{\circledR}$ has a function named sgolayfilt in the Signal Processing Toolbox. The output of a SG filter can be obtained by specifying the data vector (x), polynomial order (k) and frame length (f, which must be odd).

The moving average filter is designed as described earlier (eq. 2) using simple arithmetic operations. It is just a simple averaging operation over few points. Any output point $y[i]$ is obtained by performing moving average on input points around $x[i]$. The input points from $j=-(M-1) / 2$ to $(M-1) / 2$ are added for $x[i+j]$ and then averaged by dividing by $M$, where $M$ is the number of points used in moving average. The $\mathrm{m}$ file is later applied to implement the proposed method. The algorithm for the proposed method can be implemented using the following steps as described below:

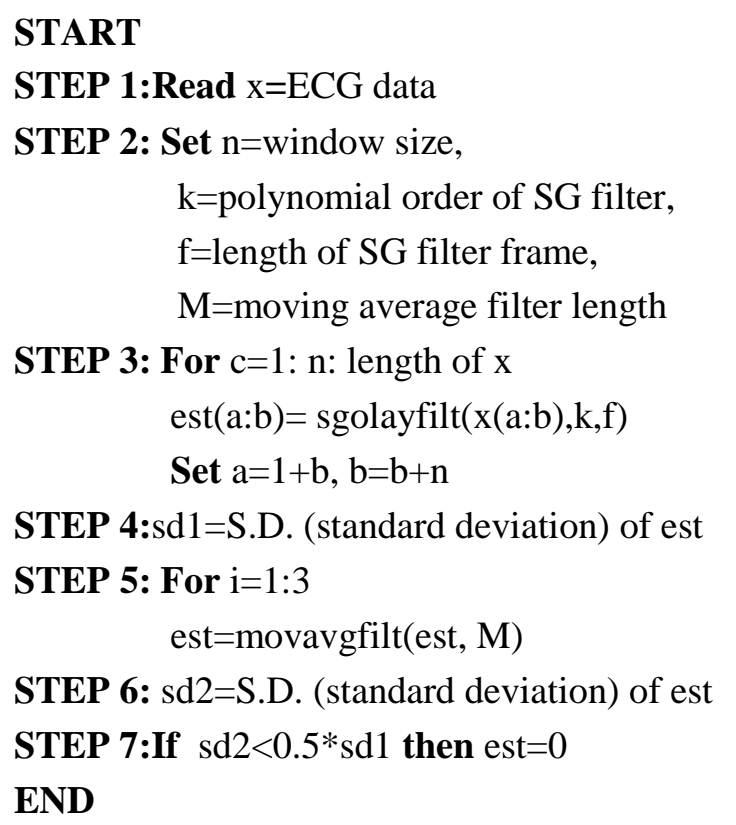

Corrected ECG signal: The estimated BW signal (est) and the original ECG data are of same length and sampling frequency. Thus simply subtracting the estimated BW from the contaminated ECG signal would result in a corrected ECG signal. The estimated BW is approximated in a way that during the process the original ECG signal remains as it is, not affecting its higher frequency components. After subtraction, the processed ECG signal might contain some minor distortions at window edges, which is extensively discussed in the next section. 


\section{RESULTS \& ANALYSIS}

The ECG signal used for analysis is taken from the MIT-BIH standard database available online at physionet.org (Goldberger et al., 2000). The signal that is taken to analyze is taken from the MIT-BIH normal sinus rhythm database and the particular signal is numbered 16265. A 10 second sample is taken from the signal with sampling frequency of $128 \mathrm{~Hz}$. The signal has two channels, only the first channel data is used for analyzing.

Assigning values to parameters in method: The proposed method requires some parameter values to be assigned prior to the processing of the ECG signal. The ECG signal used from the Physionet database is 10 seconds long and sampled at $128 \mathrm{~Hz}$, thus contains 1280 samples. The window size (n) is selected as 64 samples as it would resemble $1 / 4$ cycle of a $0.5 \mathrm{~Hz} \mathrm{BW}$, as BW frequency is usually less than 0.5 $\mathrm{Hz}$ (Macfarlane, 2011). Then selecting polynomial order (k) of 1 in the SG filter would give a linear approximation in that window. The frame length (f) of SG filter should be odd and to eliminate any effect of $R$ peaks the selected value of $f$ is 63 (almost equal to window size). It would then only follow the baseline trend. The length $(\mathrm{M})$ of moving average filter if selected as $5 \%$ of the original data length, then gives a good smoothing along with preserving the trend. Thus for a data length of 1280 , the value of $\mathrm{M}$ is selected 63 (must be odd). After setting all these parameters the proposed method is used to estimate the BW and remove it from ECG signal.

Estimation and removal of baseline wander: Different type of BW is induced and the performance of the method is analyzed. It is done for six different types of induced BW.

(a) Estimation for $0.1 \mathrm{~Hz}$ BW: First of all a simple sine wave of $0.1 \mathrm{~Hz}$ is used as normal respiration might induce such kind of artifact. It is a then added to the normal ECG signal and the subsequent BW induced ECG signal is processed using the method. Fig. 2 shows the processing. It shows step by step processing and comparison of estimated $\mathrm{BW}$ with original $\mathrm{BW}$, also processed and clean ECG.

(b) Estimation for BW of mixed frequency: The next induced BW is of a mixture of sinewave of 0.1 $\mathrm{Hz}$ and $0.5 \mathrm{~Hz}$. Fig. 3 shows the processing method.

(c) Estimation for localized BW: Now the BW induced is a localized sine wave of $0.5 \mathrm{~Hz}$. It is a single cycle of wave between samples (640 and 896).The effect is demonstrated in fig. 4.

(d) Estimation for square wave BW: For a BW such as an abrupt shift can be resembled with a square wave. The processing of ECG signal with such baseline shift is shown in fig 5 .

(e) Estimation for triangular sawtooth BW: Another standard BW testing signal is a triangular sawtooth wave. Fig. 6 shows the processing of ECG signal for such kind of induced BW.

(f) Estimation for real BW: Focus can be now given on real BW that can be present in ECG signal.

Such a signal is used from the MIT-BIH database (Goldberger et al., 2000). A real BW induced ECG

signal correction is shown in fig. 7.

Comparative analysis with usual polynomial fitting method: The proposed method is compared with usual polynomial fitting method, where polynomial fitting is applied over the whole ECG signal and the best fitted order has to be selected by experimentation. BW signals those were used in previous section, all would be compared here, thus presenting a comparative analysis of estimation of BW.

(a)Analysis for BW of $0.1 \mathrm{~Hz}$ : Fig. 8a shows estimation of baseline using both SG filter based method and polynomial fitting. It can be seen from the figure that both the methods approximates baseline similarly. SG filter based method requires only polynomial order of 1 . The polynomial fitting in this case requires $3^{\text {rd }}$ order. This higher order requirement problem would be more profound when BW would be of higher frequency and more irreugular signals would appear. 

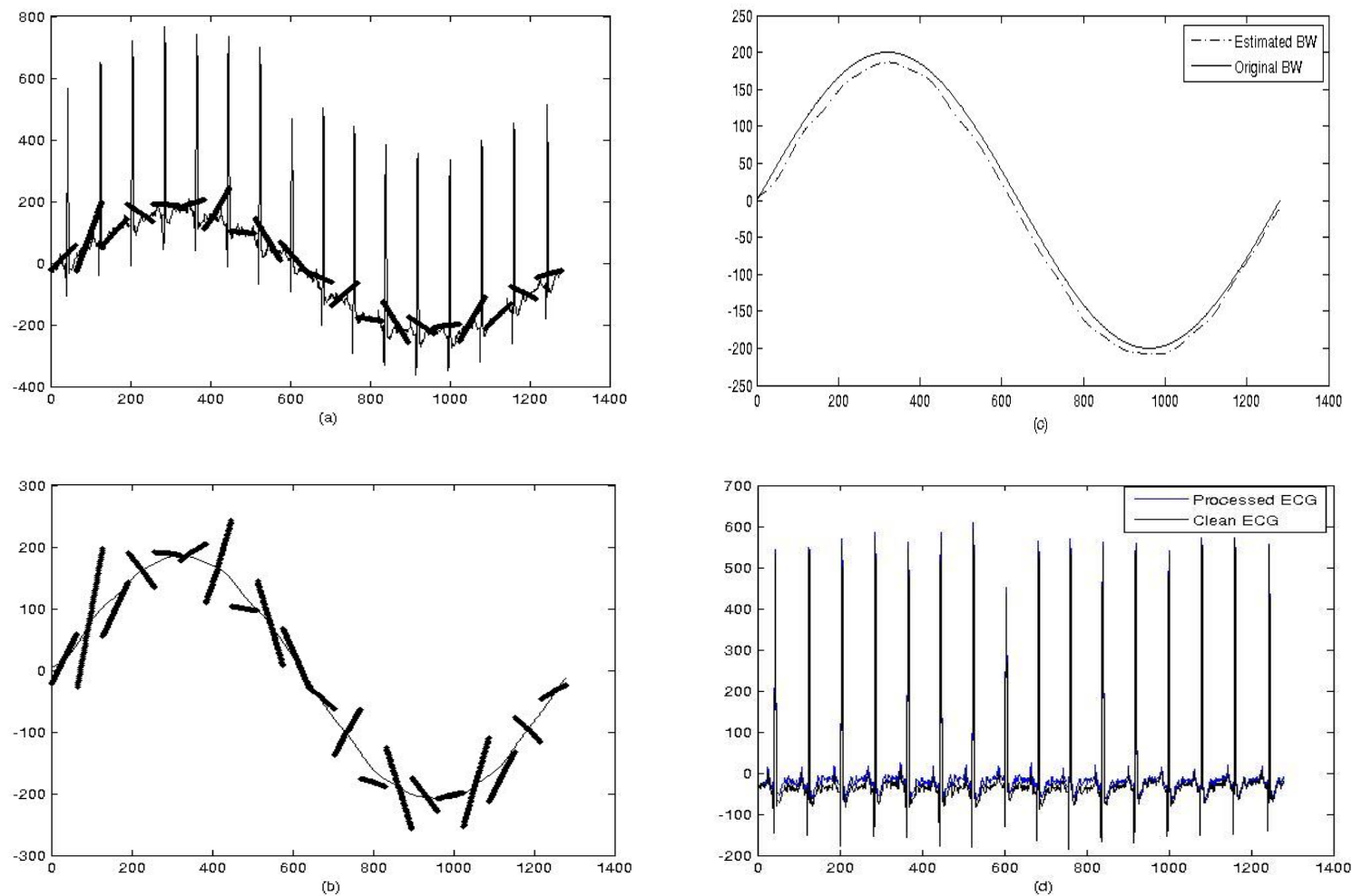

Fig. 2: (a) SG filtering on BW ( $0.1 \mathrm{~Hz}$ sinewave) induced ECG (b) After moving average filtered stage (c) BW estimation (d) Comparison of clean and processed ECG
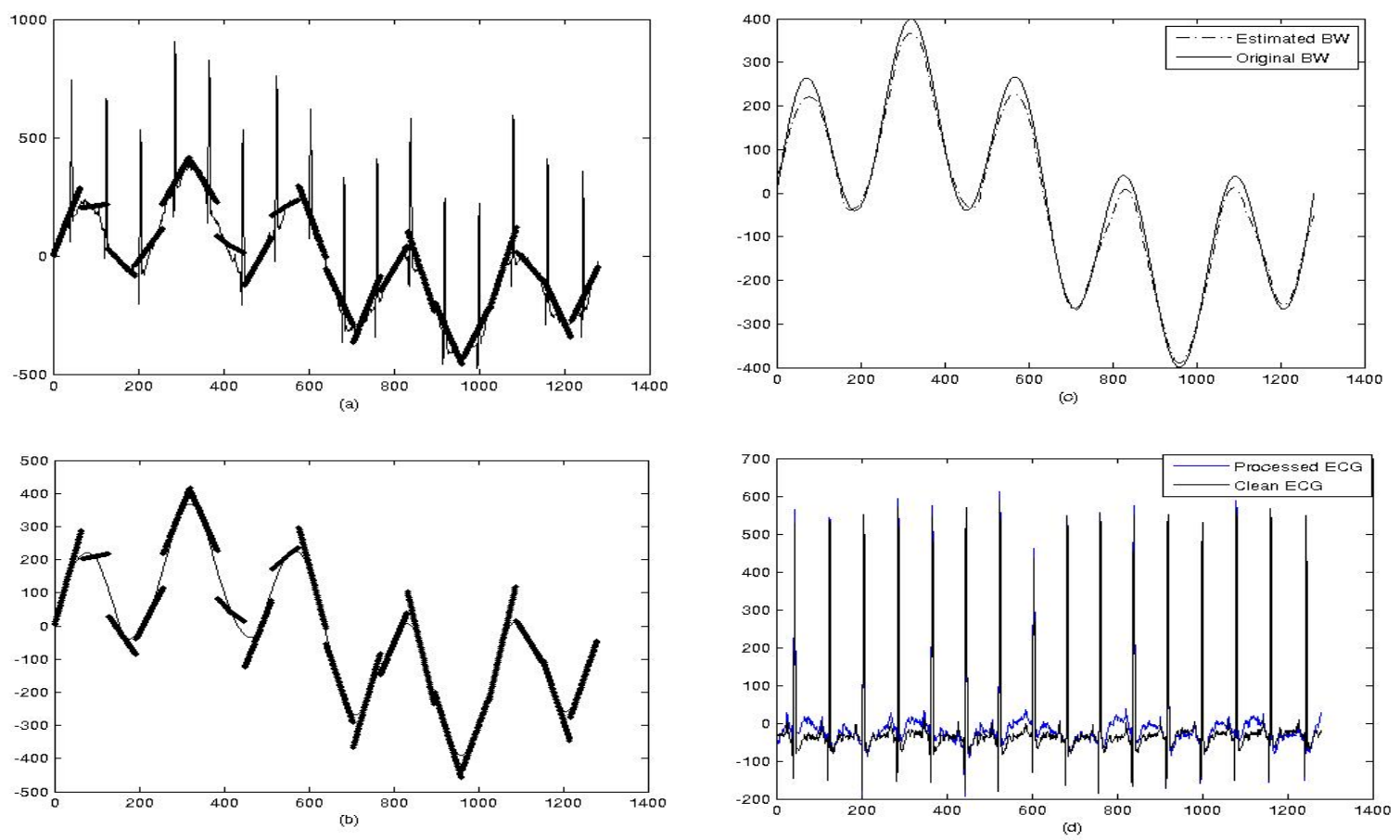

Fig. 3:(a) SG filtering on BW (mixed sinewave) induced ECG (b) After moving average filtered stage (c) BW estimation (d) Comparison of clean and processed ECG 

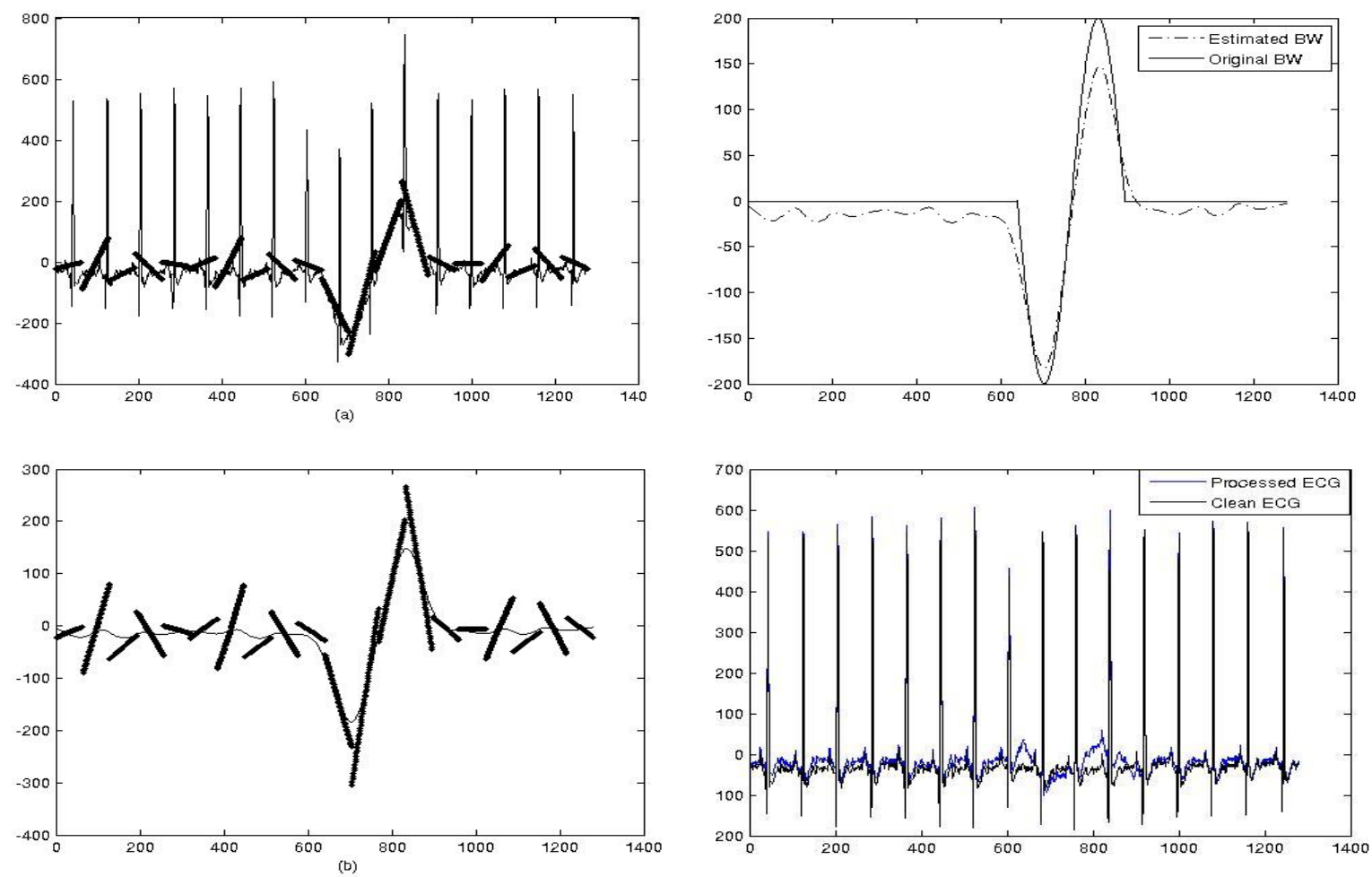

Fig. 4: (a) SG filtering on localized BW (0.5 Hz sinewave) induced ECG (b) After moving average filtered stage (c) BW estimation (d) Comparison of clean and processed ECG
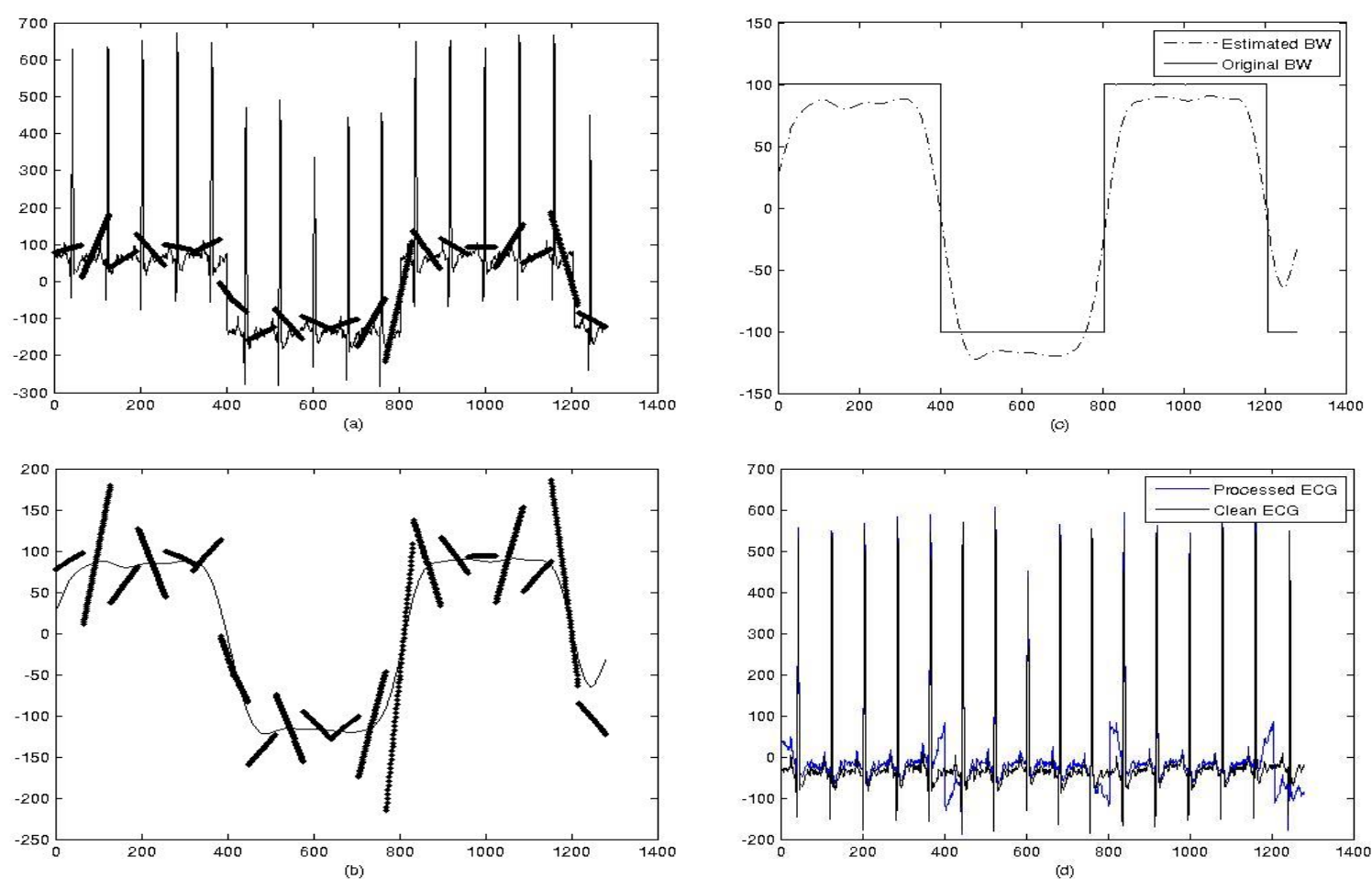

Fig. 5: (a) SG filtering on BW (square wave) induced ECG (b) After moving average filtered stage (c) BW estimation (d) Comparison of clean and processed ECG 

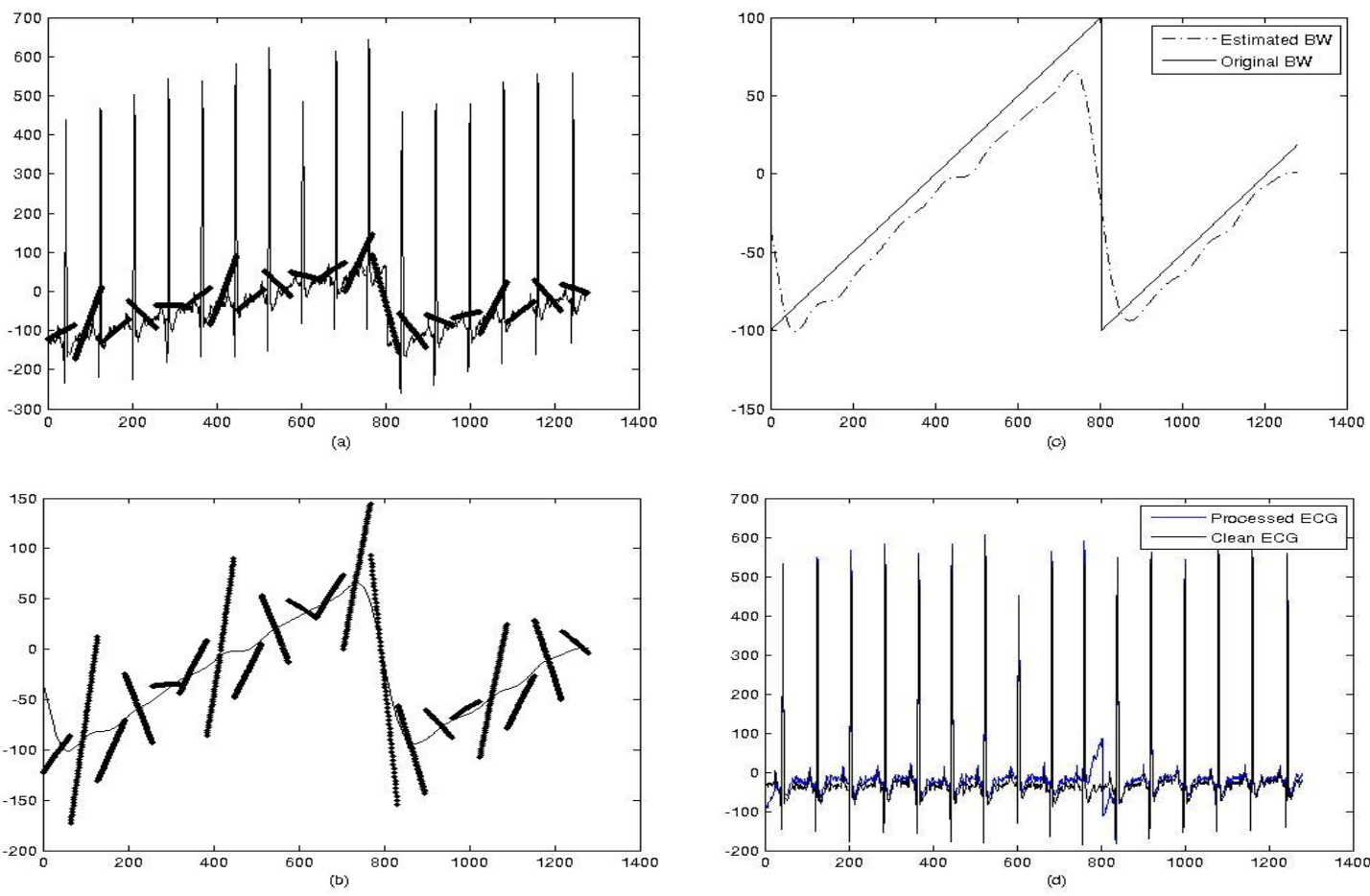

Fig 6: (a) SG filtering on BW (sawtooth wave) induced ECG (b) After moving average filtered stage (c) BW estimation (d) Comparison of clean and processed ECG
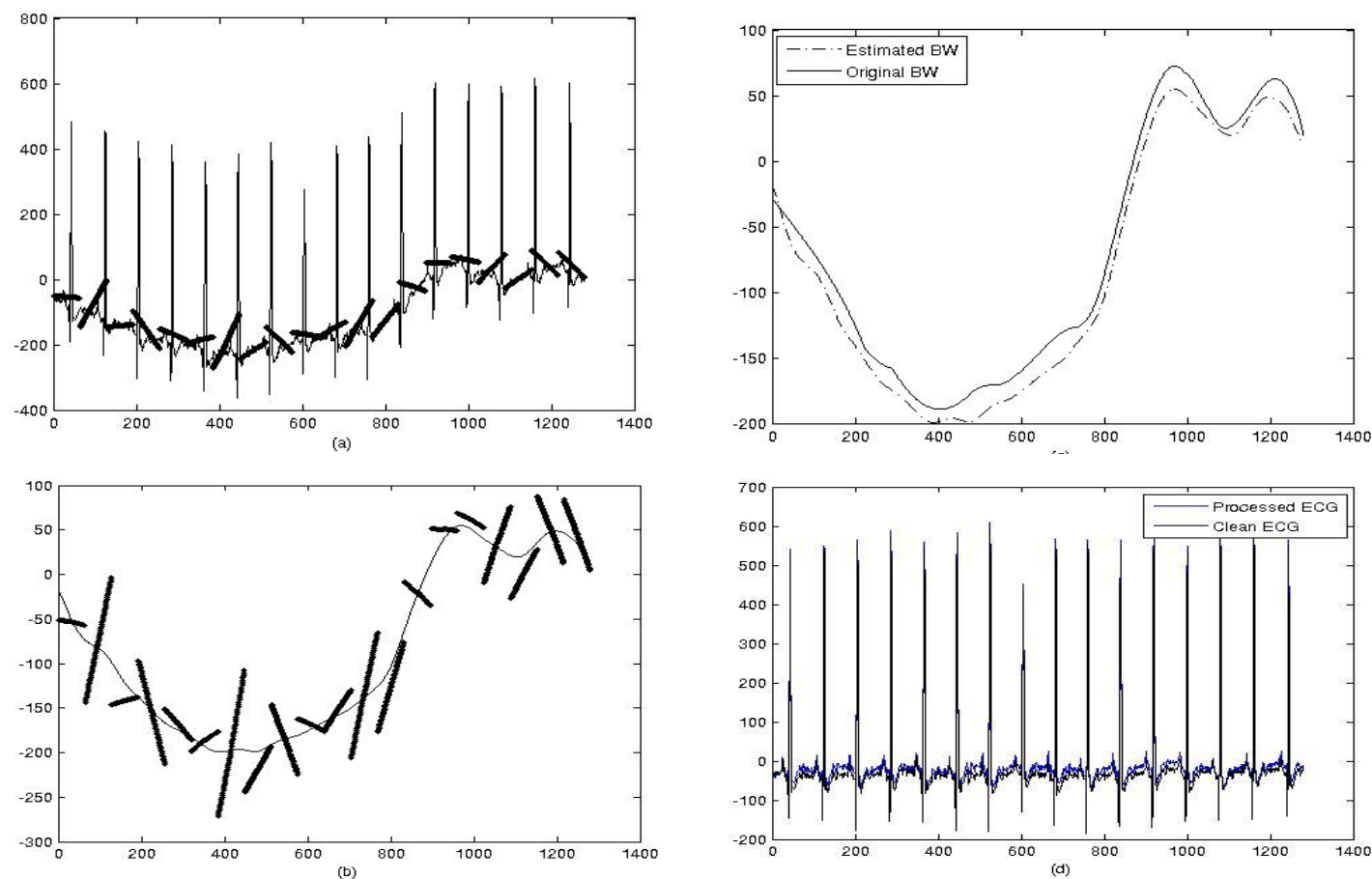

Fig 7: (a) SG filtering on real BW induced ECG (b) After moving average filtered stage (c) BW estimation (d) Comparison of clean and processed ECG 
(b) Analysis for BW of mixed frequency: For BW of mixed frequency $(0.1 \mathrm{~Hz}$ and $0.5 \mathrm{~Hz})$ comparative analysis is shown in fig. 8b. In this case polynomial order required for minimal approximation is 15 ; even higher order could better the approximation of polynomial fitting method. At this point the SG filter based method is far better than usual polynomial fitting method, as order required is only 1 .

(c) Analysis for localized BW: A localized BW is compared in this case. To get a similar estimation as SG filter method the polynomial filter requires $20^{\text {th }}$ order. As order is so high there is severe ringing effect on the isoelectric line. The SG filter method suffers some peak flattening as a result of smoothing effect, but estimates the BW far easily than polynomial method. There is also some ringing effect present but that is minimal compared to the polynomial method. The analysis shown in fig. $8 \mathrm{c}$

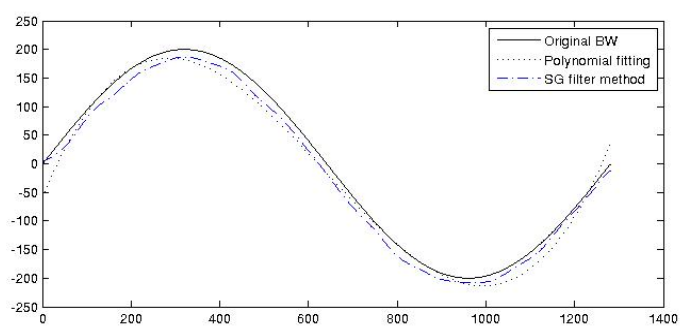

(a)

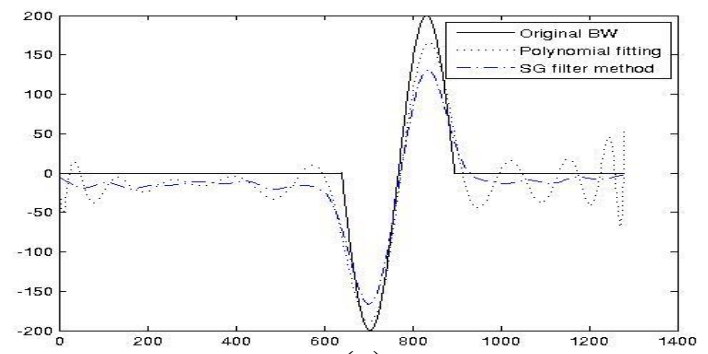

(c)

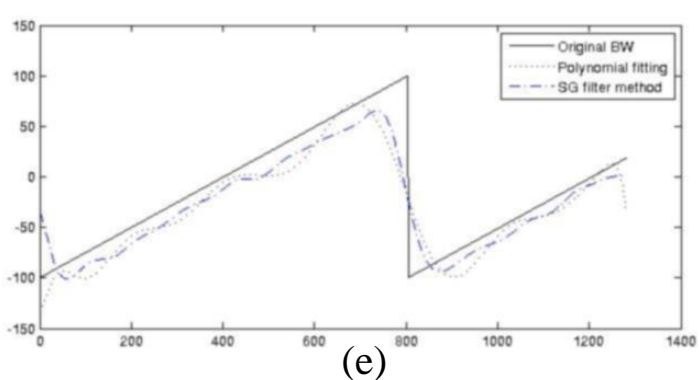

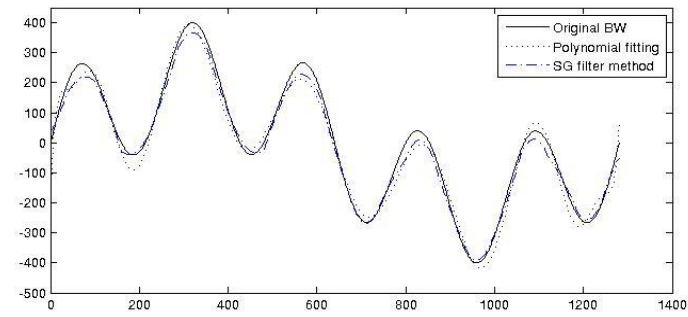

(b)

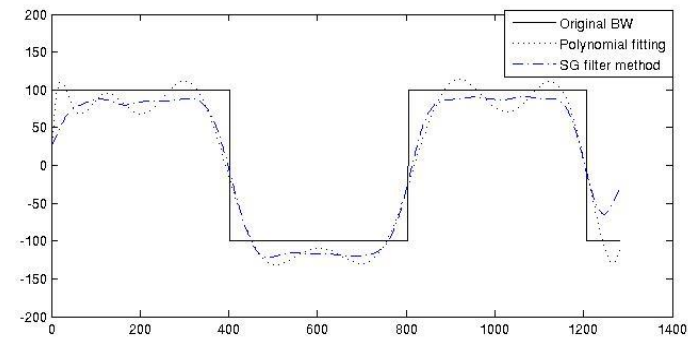

(d)

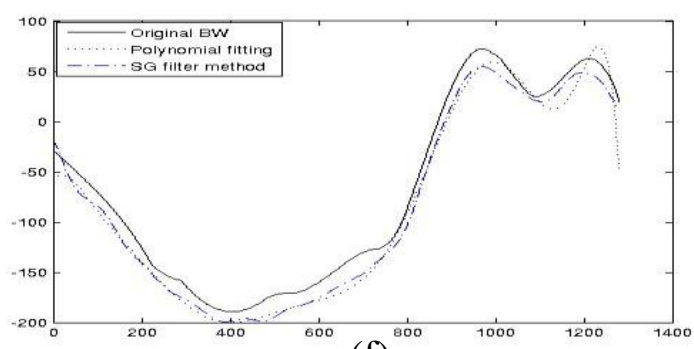

(f)

Fig. 8:Comparative analysis of polynomial fitting and proposed SG filter based method (a) For 0.1 $\mathrm{Hz}$ BW (b) For mixed frequency $(0.1 \mathrm{~Hz}$ and $0.5 \mathrm{~Hz}) \mathrm{BW}$ (c) For localized BW $(0.5 \mathrm{~Hz})$ (d) For square wave BW (e) For triangular sawtooth BW (f) For a real BW

(d) Analysis for square wave BW: The sharp edge of the square wave is not fully predicted by any of the two methods but the SG filter based method has lesser ringing and follows the original wave closely. The polynomial fitting requires at least $15^{\text {th }}$ order fitting to estimate the square wave along with significant ripples. So, in this case too, the performance of the proposed method is better. The necessary analysis is shown in fig. $8 \mathrm{~d}$.

(e) Analysis for triangular sawtooth BW: A comparison of the two methods for a triangular sawtooth wave BW is shown in fig. 8e. The polynomial order required for usual method is at least 15 and also 
shows more ringing than the SG filter based method. The SG filter based performs better in this instance also.

(f)Analysis for real BW: Attention is now turned to a typical real BW. The analysis is shown in fig. 8f. The SG filter method estimates and follows the BW more accurately and closely than the polynomial fitting method. Also the polynomial fitting requires at least $10^{\text {th }}$ order to approximate the BW closely. Thus in this case also, the proposed method supersedes.

Correlation coefficient of estimated BW: Correlation coefficient is a statistical measure that shows the linear relationship between a pair of variables. The value of correlation coefficient lies between -1 to +1 . Values close to +1 indicate that the variables have a positive linear relationship. Such value also is a measure of how closely a variable is following the other.

Table 1 shows correlation coefficient of estimated BW (for both proposed method and polynomial fitting) with original BW. Estimated BW from both methods closely follows the original BW. The proposed SG filter method has slightly better correlation coefficient than the polynomial fitting method. So, statistically the proposed method performs better too.

Table 1: Correlation coefficient of estimated BW

\begin{tabular}{|l|l|l|}
\hline $\begin{array}{l}\text { Type of Baseline } \\
\text { Wander }\end{array}$ & $\begin{array}{l}\text { Correlation Coefficient of } \\
\text { estimated BW (SG filter } \\
\text { method) }\end{array}$ & $\begin{array}{l}\text { Correlation Coefficient of estimated } \\
\text { BW (Polynomial fitting) }\end{array}$ \\
\hline (a) $0.1 \mathrm{~Hz} \mathrm{BW}$ & 0.9996 & 0.9954 \\
\hline $\begin{array}{l}\text { (b) mixed frequency } \\
\text { (0.1 Hz and 0.5 } \\
\text { Hz) BW }\end{array}$ & 0.9888 & 0.9887 \\
\hline $\begin{array}{c}\text { (c) localized BW } \\
(0.5 \mathrm{~Hz})\end{array}$ & 0.9730 & 0.9467 \\
\hline $\begin{array}{c}\text { (d) square wave BW } \\
\text { sawtooth BW }\end{array}$ & 0.9511 & 0.9479 \\
\hline (e) triangular & 0.9457 & 0.9239 \\
\hline (f) real BW & 0.9971 & 0.9916 \\
\hline
\end{tabular}

\section{DISCUSSIONS}

In all cases the proposed method has worked better than the usual polynomial fitting method. It is also verified by better correlation coefficient of the estimated BW. The polynomial fitting method requires very high order in most of the cases. For very low frequency BW, polynomial fitting can estimate it with low polynomial order. But for higher frequency cases, standard testing waveforms (square wave and saw tooth wave) and real BW it always required order in the range of 10-20. Different type of BW requires different polynomial order, so it is a challenge to set the order globally or make an order 
selection algorithm. On the other hand proposed method always estimated the BW better, just by using combination of SG filter of order 1 and moving average filter. Due to use of higher orders the polynomial fitting method has had always significant ripples on the isoelectric line and the estimated BW. Though the proposed method also shows some ringing on the isoelectric line, it does not affect the ECG signal severely as higher order polynomial fitting does. Along the sharp edges of BW, in case of some abrupt shift, the proposed method follows the trend better than usual polynomial fitting. The SG filter does not simply extract the trend based on moving average filtering. Rather it takes into account the order of that polynomial fitting. In this way it can make better approximation. In the proposed method, the polynomial order is kept always 1 but window size is selected in such a way that the fitting would be appropriate. Thus, it keeps the complexity of the method at minimal level, as increasing order would make the calculations complex. Moreover, using the lowest possible order ensures less ringing or ripple on the isoelectric line.

\section{LIMITATIONS}

The proposed method has limitations which can restrict its efficiency in some cases. Any moving average filter would flatten waveform peaks during the course of smoothing operation. As in this method SG filter is used and also moving average filtering is applied, there are some cases of minor peak flattening observed in the analysis, which is expected and arises from the segmentation and applying joining method after SG filter. Even though the polynomial order of SG filter was set to 1, due to windowing and later moving averaging, there arise some ripples in the estimated BW, which is unwanted. Another limitation that can be mentioned of is the inability to detect sharp edges of BW accurately. Though it follows the trend quite accurately, the edges are smoothed out due to moving average filtering effect. But the precision of this proposed method is still superior to the conventional methods like polynomial fitting.

\section{FUTURE WORK}

The scope of future work lies in overcoming the limitations of the method. Algorithm which takes into consideration the peak flattening and somehow compensates for it can be useful if implemented. The ringing or rippling effect could be eliminated if some decision rule is set in every window to reject the estimation if no $\mathrm{BW}$ is present in that particular window. The detection of sharp edges is tricky as any moving average filtering smooths the edges. If the detected edges could be preserved somehow, then it could be possible to estimate this type of BW more accurately. Also automatically selecting the window size, order or filter frame length based on some characteristics of ECG signal could make the method more adaptive. Overall, despite all limitations it can be stated that the proposed method works fairly well in estimating and removing BW from ECG signal.

\section{ACKNOWLEDGEMENT}

International Science Programme of Uppsala University, Sweden, for part financial support.

\section{REFERENCES}

Akay, M. (2006).Wiley encyclopedia of biomedical engineering. Hoboken, N.J.: Wiley- InterScience.

Badilini, F., Moss, A. and Titlebaum, E. (1991).Cubic spline baseline estimation in ambulatory ECG recordings for the measurement of ST segment displacements.Proceedings of the Annual Internatlonal Conference of the IEEE Engineering in Medicine and Biology Society, 13(2), pp.584-585.

Bailey, J. (2004). The triangular wave test for electrocardiographic devices: A historical perspective. Journal of Electrocardiology, 37, pp.71-73. 
Bailey, J., Berson, A., Garson, A., Horan, L., Macfarlane, P., Mortara, D. and Zywietz, C. (1990). Recommendations for standardization and specifications in automated electrocardiography: bandwidth and digital signal processing. A report for health professionals by an ad hoc writing group of the Committee on Electrocardiography and Cardiac Electrophysiology of the Council on Clinical Cardiology, American Heart Association.Circulation, 81(2), pp.730-739.

Blanco-Velasco, M., Weng, B. and Barner, K. (2008). ECG signal denoising and baseline wander correction based on the empirical mode decomposition. Computers in Biology and Medicine, 38(1), pp.1-13.

de Pinto, V. (1992). Filters for the reduction of baseline wander and muscle artifact in the ECG. Journal of Electrocardiology, 25, pp.40-48.

Frankel, R., Pottala, E., Bowser, R. and Bailey, J. (1991). A filter to suppress ECG baseline wander and preserve ST-segment accuracy in a real-time environment. Journal of Electrocardiology, 24(4), pp.315-323.

Froning, J., Olson, M. and Froelicher, V. (1988). Problems and limitations of ECG baseline estimation and removal using a cubic spline technique during exercise ECG testing: Recommendations for proper implementation. Journal of Electrocardiology, 21, pp.149-157.

Goldberger, A., Amaral, L., Glass, L., Hausdorff, J., Ivanov, P., Mark, R., Mietus, J., Moody, G., Peng, C. and Stanley, H. (2000).PhysioBank, PhysioToolkit, and PhysioNet : Components of a New Research Resource for Complex Physiologic Signals. Circulation, 101(23), pp.e215-e220.

Hargittai, S. (2005).Savitzky-Golay Least-Squares Polynomial Filters in ECG Signal Processing. Computers in Cardiology, (32), pp.763-766.

Huang, N., Shen, Z., Long, S., Wu, M., Shih, H., Zheng, Q., Yen, N., Tung, C. and Liu, H. (1998). The empirical mode decomposition and the Hilbert spectrum for nonlinear and non-stationary time series analysis.Proceedings of the Royal Society A: Mathematical, Physical and Engineering Sciences, 454(1971), pp.903-995.

Jane, R., Laguna, P., Thakor, N. and Caminal, P. (1992). Adaptive baseline wander removal in the ECG: comparative analysis with cubic spline technique. Computers in Cardiology.

Macfarlane, P. (2011). Comprehensive electrocardiology. New York: Springer.

Meyer, C. and Keiser, H. (1977).Electrocardiogram baseline noise estimation and removal using cubic splines and state-space computation techniques.Computers and Biomedical Research, 10(5), pp.459-470.

Orfanidis, S. (1996). Introduction to signal processing. Englewood Cliffs, N.J.: Prentice Hall.

Park, K., Lee, K. and Yoon, H. (1998).Application of a wavelet adaptive filter to minimise distortion of the STsegment.Medical \& Biological Engineering \& Computing, 36(5), pp.581-586.

Pottala, E., Bailey, J., Horton, M. and Gradwohl, J. (1990). Suppression of baseline wander in the ECG Using a bilinearly transformed, null-phase filter. Journal of Electrocardiology, 22, pp.243-247.

Savitzky, A. and Golay, M. (1964). Smoothing and Differentiation of Data by Simplified Least Squares Procedures. Analytical Chemistry, 36(8), pp.1627-1639.

Schafer, R. (2011). What Is a Savitzky-Golay Filter? [Lecture Notes].IEEE Signal Process.Mag., 28(4), pp.111117.

Smith, S. (2002). The scientist and engineer's guide to digital signal processing. [San Diego, Calif.]: California Technical Pub.

Van Alste, J. and Schilder, T. (1985).Removal of Base-Line Wander and Power-Line Interference from the ECG by an Efficient FIR Filter with a Reduced Number of Taps.IEEE Transactions on Biomedical Engineering, BME-32(12), pp.1052-1060.

Webster, J. (1988). Encyclopedia of medical devices and instrumentation. - Volume 3. New York,N.Y.: Wiley.

Zheng, L., Lall, C. and Chen, Y. (2012). Ling Zheng, Lall, C., Yu Chen, Low-Distortion Baseline Removal Algorithm for Electrocardiogram Signals. Computers in Cardiology, (39), pp.769-772. 